\title{
Rotational dynamics and friction in double-walled carbon nanotubes
}

\author{
J. Servantie and P. Gaspard \\ Center for Nonlinear Phenomena and Complex Systems \\ Université Libre de Bruxelles, Code Postal 231, Campus Plaine, 1050 Brussels, Belgium.
}

(Dated: June 12, 2018)

\begin{abstract}
We report a study of the rotational dynamics in double-walled nanotubes using molecular dynamics simulations and a simple analytical model reproducing very well the observations. We show that the dynamic friction is linear in the angular velocity for a wide range of values. The molecular dynamics simulations show that for large enough systems the relaxation time takes a constant value depending only on the interlayer spacing and temperature. Moreover, the friction force increases linearly with contact area, and the relaxation time decreases with the temperature with a power law of exponent $-1.53 \pm 0.04$.

PACS numbers: 68.35.Af; 85.35.Kt
\end{abstract}

Since the pioneering work of Iijima on nanotubes [1], research on their electronic and mechanic properties has been rapidly growing. Very high electric and thermal conductivities were observed and the nanotubes were shown to be as stiff as diamond in their axial direction with Young modulus of the order of TPa. These observations make the nanotubes promising candidates for nanoelectrical and nano-mechanical devices. The experiments on the translational motion in multi-walled nanotubes of Cumings and Zettl [2] and Yu et al. [3] motivated the study of these possible oscillators. Many numerical studies were performed to characterize the translational motion of double-walled nanotubes [4, [5, 6, 7, 8]. On the other hand, experiments were also carried out to study the rotational motion of concentric nanotubes. Indeed, Fennimore et al. [9] and Bourlon et al. 10 built actuators based on multi-walled nanotubes. As for the translational oscillatory motion, no wear or fatigue was observed in the rotation. Recently, Král and Sadeghpour 11] proposed to spin nanotubes with circularly polarized light, further motivating the use of nanotubes as possible axis of rotation at the nanoscale. However, few systematic analytical and numerical studies of rotation have been done, except the work of Zhang et al. 12] where nonequilibrium molecular dynamics simulations were carried out to calculate the energy dissipation rate during the rotational motion in the presence of external thermostat. Nevertheless, the friction between two carbon nanotubes which are rotating one with respect to the other has not yet been understood and characterized.

The purpose of the present Letter is to study the rotational dynamics of a double-walled nanotube (DWNT) by molecular dynamics simulations with the conservative Hamiltonian mechanics in order to avoid additional sources of dissipation and precisely characterize the rotational friction. Since friction is related to the fluctuations of the many vibrational degrees of freedom, we set up a Langevin-type stochastic model and validate it by molecular dynamics simulations of typical DWNTs. This shows that dynamic friction is proportional to the angular velocity up to high values, increases linearly with contact area, and as a power law of exponent $1.53 \pm 0.04$ with temperature.

We consider a DWNT with free ends in vacuum. The Hamiltonian of the system of two concentric nanotubes is the same as in our previous studies for the translational motion [4, 5]. The intratube interactions are modeled by the Tersoff-Brenner potential with the set of parameters of Table III in Ref. 13] and the intertube interactions by a $6-12$ Lennard-Jones potential used for carbon material simulations [14]. The DWNT is long enough so that the inner and outer nanotubes essentially rotate around a common axis with the angular velocities $\omega_{1}$ and $\omega_{2}$, respectively. Their angular momenta are given by $L_{a}=I_{a} \omega_{a}$ in terms of the moments of inertia $I_{a}$ $(a=1,2)$. The equations of motion of this model are given by

$$
\frac{d L_{a}}{d t}=I_{a} \frac{d \omega_{a}}{d t}=N_{a} \quad(a=1,2)
$$

with the torques $N_{1}=-N_{2}$ in order to conserve the total angular momentum $L_{0}=L_{1}+L_{2}$. We can introduce the angular velocity of the center of inertia $\Omega=\left(I_{1} \omega_{1}+\right.$ $\left.I_{2} \omega_{2}\right) /\left(I_{1}+I_{2}\right)=L_{0} /\left(I_{1}+I_{2}\right)$ and the relative angular velocity $\omega=\omega_{1}-\omega_{2}$ so that the equations of motion become $d \omega / d t=N_{1} / I$ and $d \Omega / d t=0$ with the relative moment of inertia $I=I_{1} I_{2} /\left(I_{1}+I_{2}\right)$. The total rotational kinetic energy of the nanotubes is given by

$$
T_{\mathrm{r}}=\frac{1}{2} I_{1} \omega_{1}^{2}+\frac{1}{2} I_{2} \omega_{2}^{2}=\frac{1}{2}\left(I_{1}+I_{2}\right) \Omega^{2}+\frac{1}{2} I \omega^{2}
$$

The corrugation against the rotational motion is very small compared to the thermal energy. Indeed, for the two DWNTs considered in this Letter, namely $(4,4) @(9,9)$ and $(7,0) @(9,9)$, and the 6-12 Lennard-Jones potential we use [14], we find a corrugation against rotation of respectively $0.00992 \mathrm{meV} /$ atom and 0.00548 $\mathrm{meV} /$ atom. These values are of the same order as the ones of Zhang et al. who used the Kolmogorov and Crespi potential 15]. Even for temperatures as low as $100 \mathrm{~K}$ 
( $8.6 \mathrm{meV})$, thermal energy is much larger than corrugation energy against rotation. This observation allows us to neglect the mean static torque due to the corrugation in the equation of motion of the system. However, in order to model the rotational dynamics of the DWNT one should take into account dynamic friction. If the angular velocity is small enough, we expect that the friction torque is linear in the angular velocity in analogy with the case of the translational motion [4, 5]. Indeed, the rotation of a nanotube inside another one is comparable to the the sliding friction of Xenon film on a silver surface considered by Daly and Krim [16] and Tomassone et al. 17] who showed that, in their system, friction is dominated by phonon excitations and that dynamic friction is proportional to the sliding velocity. Accordingly, we model our system by a Langevin-type equation without potential,

$$
I \frac{d \omega}{d t}=-\chi \omega+N_{\text {fluct }}(t)
$$

where $\chi$ is a damping coefficient and $N_{\text {fluct }}(t)$ a fluctuating torque of zero mean value and correlation function $\left\langle N_{\text {fluct }}(t) N_{\text {fluct }}\left(t^{\prime}\right)\right\rangle \simeq 2 \chi k_{\mathrm{B}} T \delta\left(t-t^{\prime}\right)$ in terms of the temperature $T$ and Boltzmann's constant $k_{\mathrm{B}}$. This is justified because the correlation time is of the order of the inverse of the Debye frequency (about $50 \mathrm{fs}$ ) as for the translational motion [4]. Since we observe relaxation times of the order of the nanosecond, one can assume this Markovian form for the evolution equation. We notice that the variations of the moments of inertia are neglected in Eq.(3).

A molecular dynamics simulation can be carried out by giving an initial angular momentum $L_{0}=I_{1} \omega_{0}$ to the inner tube $(a=1)$ and a zero one to the outer tube. After the relaxation time $\tau=I / \chi$, the system reaches a state of equilibrium in which both tubes rotate with the same angular frequency, $\left\langle\omega_{1}\right\rangle_{\text {eq }}=\left\langle\omega_{2}\right\rangle_{\text {eq. }}$. This numerical observation is in agreement with the consequence of Eq. (3) that the mean relative angular velocity vanishes at equilibrium. Using the conservation of total angular momentum, the equilibrium values of the angular momenta are thus given by $\left\langle L_{a}\right\rangle_{\text {eq }}=L_{0} I_{a} /\left(I_{1}+I_{2}\right)$ for $a=1,2$. At equilibrium, the fluctuations of the angular velocity have the variance $\left\langle\omega^{2}\right\rangle_{\mathrm{eq}}=k_{\mathrm{B}} T / I$. For times longer than the relaxation $t \gg I / \chi$, the relative angle $\theta$ performs a diffusive random rotation

$$
\left\langle\theta^{2}(t)\right\rangle \simeq 2 D(t-\tau)
$$

with the diffusion coefficient $D=k_{\mathrm{B}} T / \chi$. Moreover, the angular velocity autocorrelation function is given by

$$
\left\langle\omega(t) \omega\left(t^{\prime}\right)\right\rangle_{\mathrm{eq}}=\frac{k_{\mathrm{B}} T}{I} e^{-\lambda\left(t-t^{\prime}\right)}
$$

where we defined $\lambda=\chi / I=1 / \tau$. If a relative angular velocity $\omega_{0}$ is initially given to the system, Eq. (3) shows that it decays on average according to

$$
\langle\omega(t)\rangle=\omega_{0} e^{-\lambda t}
$$

and the total rotational kinetic energy (2) as

$$
\left\langle T_{\mathrm{r}}(t)\right\rangle=\frac{T_{0}}{I_{1}+I_{2}}\left(I_{2} e^{-2 \lambda t}+I_{1}\right)+\frac{k_{\mathrm{B}} T}{2}\left(1-e^{-2 \lambda t}\right)
$$

where $T_{0}=\left\langle T_{\mathrm{r}}(0)\right\rangle$ denotes its initial value. Hence, we conclude that, in the long-time limit $t \rightarrow \infty$, the rotational kinetic energy reaches the value

$$
\left\langle T_{\mathrm{r}}(\infty)\right\rangle=\frac{L_{0}^{2}}{2\left(I_{1}+I_{2}\right)}+\frac{k_{\mathrm{B}} T}{2}
$$

We now show the results of the molecular dynamics simulations. The integration is done with a velocity Verlet scheme of time step 2 fs for temperatures up to $600 \mathrm{~K}$ and $1 \mathrm{fs}$ for larger temperatures. Angular velocity is fixed by giving a tangential velocity corresponding to $\omega_{0}$ to the velocities of each atom of the inner tube. We compute the average relative angular velocity $\langle\omega\rangle$ and rotational kinetic energy $\left\langle T_{\mathrm{r}}\right\rangle$ from the angular momenta and moments of inertia in the axial direction. The angle is then obtained by the time integral of the angular velocity.

As a first test, we estimated the maximum angular velocity the nanotube could endure before its dislocation. One can easily understand that the velocity of rotation should be smaller than the radial phonon velocity to guarantee the stability of the system. Indeed, we have calculated the critical angular velocity at which the nanotube collapses for increasing radii of the nanotube. We found out that the critical velocity $v_{\mathrm{c}}=\omega_{\mathrm{c}} R$ is constant and approximately equal to $7.98 \pm 0.80 \mathrm{~km} / \mathrm{s}$, close to the velocity $8 \mathrm{~km} / \mathrm{s}$ of radial phonons [5]. However, Zhang et al. 12] observed the collapse of their nanotube for angular velocities higher than $4.7 \mathrm{rad} / \mathrm{ps}$, corresponding to a radial velocity of approximately $3.1 \mathrm{~km} / \mathrm{s}$. This smaller value is probably due to the fact that the angular velocity was applied incrementaly to constraint atoms at the end of the tube while we give as an initial condition the angular velocity to all the atoms of the nanotube.

We apply our model to the armchair-armchair DWNT $(4,4) @(9,9)$ with both nanotubes of length $2.11 \mathrm{~nm}$ and inertia moments $I_{1}=139.11 \mathrm{amu} \mathrm{nm}{ }^{2}$ and $I_{2}=1547.9$ amu $\mathrm{nm}^{2}\left(I=127.64 \mathrm{amu} \mathrm{nm}^{2}\right)$ at room temperature $(300 \mathrm{~K})$. We depict in Fig. 1 the time evolution of the angular frequency and the rotational kinetic energy from an initial relative angular velocity of $\omega_{0}=1.07 \mathrm{rad} / \mathrm{ps}$. The relaxation time calculated from the angular velocity in Fig. 1(a) is 473 ps while the relaxation time from the energy is 243 ps which is in very good agreement with the factor 2 appearing between the decay rates in Eqs. (6) and (7). The decay of the rotational kinetic energy is well fitted by Eq. (17) with the initial kinetic energy $T_{0}=0.822 \mathrm{eV}$. On the other hand, the simulations show that the equilibrium kinetic energy takes the value 0.0816 


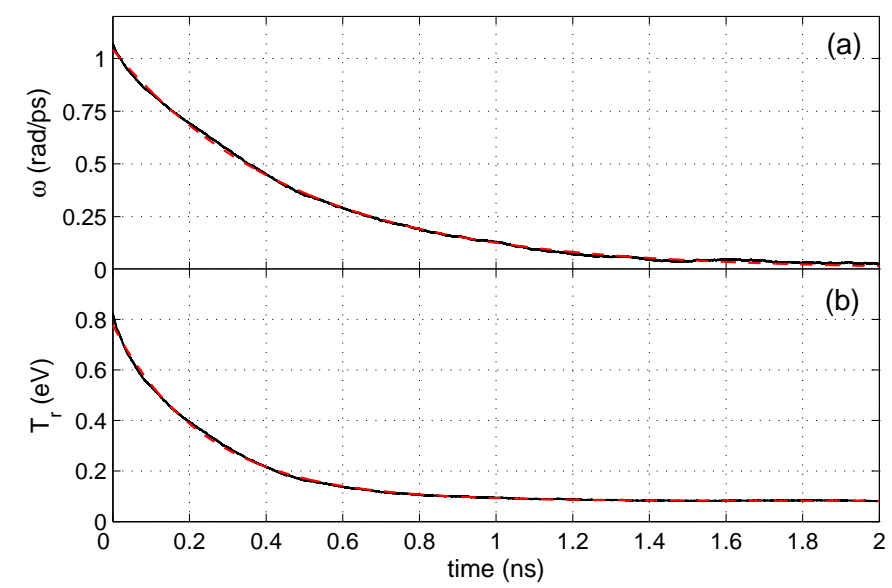

FIG. 1: (Color online) Plots of the time evolution of (a) the average relative angular velocity $\langle\omega\rangle$ and (b) the average rotational kinetic energy $\left\langle T_{\mathrm{r}}\right\rangle$ for the armchair-armchair DWNT described in the text. The dashed lines are the fits by (a) Eq. (6) and (b) Eq. (7). The averages are computed with about $10^{3}$ trajectories.

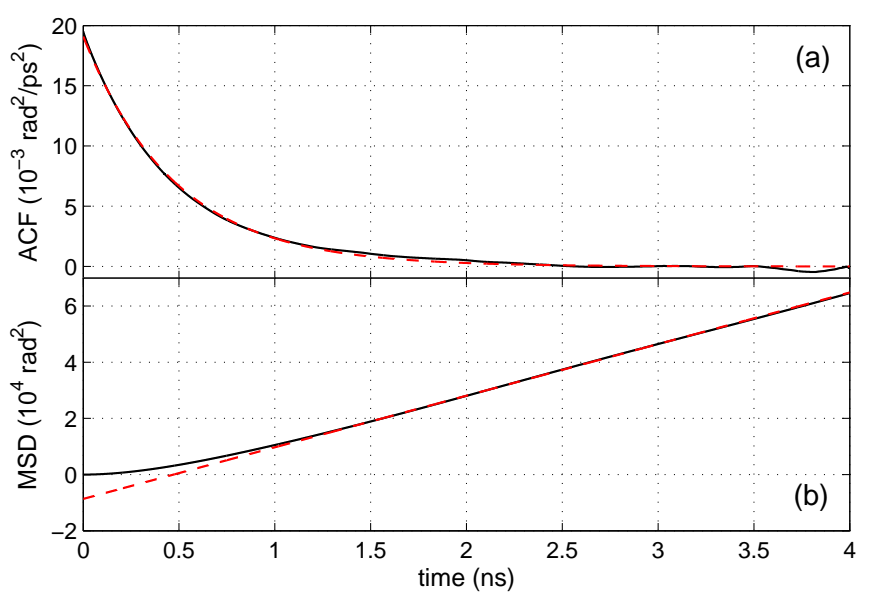

FIG. 2: (Color online) Plots of the time evolution of (a) the velocity autocorrelation function and (b) the mean square displacement of the angle for the armchair-armchair DWNT described in the text. The dashed lines are the fits by (a) Eq. (5) and (b) Eq. (4). The statistics are computed with about $10^{3}$ trajectories.

$\mathrm{eV}$, which is in excellent agreement with Eq. (8) of the model. In order to confirm the validity of the stochastic model described in this Letter, we use the same code, but with a vanishing initial angular velocity. The velocity autocorrelation function and the mean square displacement are calculated after a time of 1 ns longer than the relaxation. The results are depicted in Fig. 2 The velocity autocorrelation function is well fitted by the exponential (5D) with a relaxation time of $475.9 \mathrm{ps}$ while the linear fit (4) on the mean square displacement gives $\tau=471.3$ ps. These results are in excellent agreement with the previous simulations. The small differences are due to the statistical error, and can be reduced with longer simulation times. These simulations successfully demonstrate that the dynamic friction against the rotational motion is proportional to angular velocity in DWNTs.

We calculated the dependence of the damping coefficient on contact area at room temperature $(300 \mathrm{~K})$ for increasing lengths of our DWNTs. In order to obtain this result, we measured the relaxation time $\tau$ of the angular velocity and calculated the damping coefficient as $\chi=I / \tau$. The damping coefficient $\chi$ is depicted as a function of the length of the tubes in Fig. 3 where we see that the linear fit is in excellent agreement with the simulations. The friction force does not vanish at zero contact area because of the attractive forces between the surfaces [18]. Having obtained the dependence on length, we investigated the dependence on radius. We calculated the relaxation time for increasing radii of zigzag-armchair DWNTs. Our numerical simulation gives no systematic dependence of the relaxation time on radius except the variations due to the different interlayer spacings considered. Hence the dependence on radius of the damping coefficient comes only from the inertia moment $I$. The inertia moment of a nanotube of length $\ell$, radius $R$ and surface mass density $\sigma$ is very well approximated by $I \simeq 2 \pi \sigma \ell R^{3}$. For the hexagonal lattice, the surface mass density is $\sigma=4 m /\left(3 \sqrt{3} a_{\mathrm{CC}}^{2}\right)$ where $m=12 \mathrm{amu}$ is the atomic mass of carbon and $a_{\mathrm{CC}}=1.42 \AA$ the carboncarbon bond length. Hence, $\sigma=4.55 \mathrm{amu} / \AA^{2}$. In order to recover the experimental conditions [9, 10] where the inner ring is fixed and the outer free, we suppose that $I_{1} \rightarrow \infty$ so that the relative inertia moment is $I=I_{2}$. Since the friction torque is $N=\chi \omega$ and the angular velocity $\omega=v / R$, the friction force is given by

$$
F=\frac{N}{R}=\frac{\chi}{R^{2}} v=\frac{\sigma A}{\tau} v
$$

with the contact area $A=2 \pi \ell R$. For nanotubes long enough $\ell \gg \ell_{0}$, the relaxation time becomes independent of the length as shown in Fig. 3 and the shear stress can be written as $F / A=\sigma v / \tau_{\infty}$. As an example, the shear stress undergone by a nanotube of radius $10 \mathrm{~nm}$ with a rotational frequency of $1 \mathrm{GHz}$ is approximately $0.047 \mathrm{MPa}$. This confirms the expectation that the friction force increases linearly with the contact area $A$ [19]. Nevertheless, we notice that friction could be enhanced because of the presence of impurities and deformations due to the plate fixed to the outer tube in the experiments of Refs. [9, 10].

We have also investigated the dependence of the damping coefficient on the temperature by the method of the autocorrelation function. A smaller DWNT have been used to gain computation time, namely a $(4,4) @(9,9)$ of length $9.8 \AA$. Indeed, the relaxation time is very large for low temperature. Since the dynamic friction is due to phonon interaction in the DWNT, one expects it to increase with temperature. On the other hand, at zero 


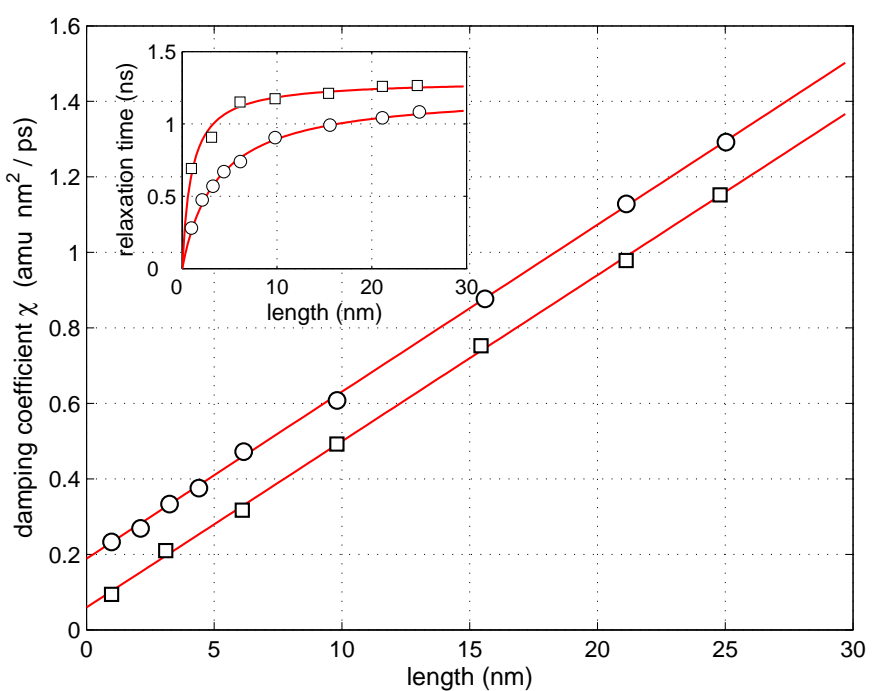

FIG. 3: (Color online) Dependence of the damping coefficient $\chi$ on length. The circles and squares are the results of the simulations for respectively the armchair-armchair DWNT $(4,4) @(9,9)$ and the zigzag-armchair DWNT $(7,0) @(9,9)$. The linear fits have the same slope $0.044 \mathrm{amu} \mathrm{nm} / \mathrm{ps}$. The inset is the relaxation time versus length $\ell$, which is well fitted by $\tau=\tau_{\infty} \ell /\left(\ell+\ell_{0}\right) . \quad \tau_{\infty}=1.22 \mathrm{~ns}$ and $\ell_{0}=3.6 \mathrm{~nm}$ for the armchair-armchair DWNT. $\tau_{\infty}=1.30 \mathrm{~ns}$ and $\ell_{0}=1.0 \mathrm{~nm}$ for the zigzag-armchair DWNT.

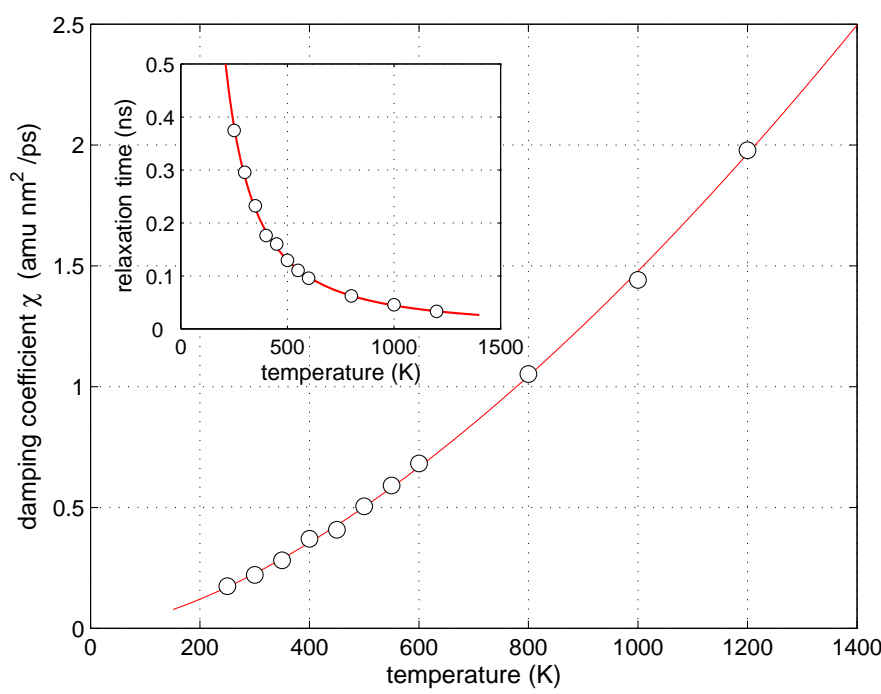

FIG. 4: (Color online) Dependence of the damping coefficient $\chi$ on temperature. The circles are the simulation results and the line is a power law fit of exponent $1.53 \pm 0.04$. The inset is the relaxation time versus temperature.

temperature, the rotational motion should become ballistic and the diffusion coefficient $D=k_{\mathrm{B}} T / \chi$ should diverge. Indeed, our results confirm these expectations since we find that the damping coefficient depends on the temperature as the power law $\chi \sim T^{\nu}$ with the exponent $\nu=1.53 \pm 0.04$, as seen in Fig. 四 Accordingly, the relaxation time for long enough DWNTs behaves as $\tau_{\infty}=\tau_{*}\left(T_{*} / T\right)^{\nu}$ where the time $\tau_{*}$ depends mainly on the interlayer spacing of the DWNT and $T_{*}$ is some reference temperature. We notice that dynamic friction may be non-vanishing at very low temperature where it is dominated by electronic interactions and impurities.

In conclusion, we proposed a stochastic model describing very well the rotational dynamics of DWNTs. We showed that dynamical friction is proportional to the angular velocity if this later is not too high. Moreover, for long enough nanotubes, the friction force is proportional to the contact area and can be characterized in terms of a relaxation time $\tau_{\infty}$ which depends on the temperature and the interlayer spacing. For higher velocities, nonMarkovian and nonlinear effects can arise resulting into an increased dissipation.

This research is financially supported by the "Communauté française de Belgique" (contract "Actions de Recherche Concertées" No. 04/09-312) and the National Fund for Scientific Research (F. N. R. S. Belgium, contract F. R. F. C. No. 2.4577.04).

[1] S. Iijima, Nature (London) 354, 56 (1991).

[2] J. Cumings and A. Zettl, Science 289, 602 (2000).

[3] M.-F. Yu, B. I. Yakobson, and R. S. Ruoff, J. Phys. Chem. B 104, 8764 (2000).

[4] J. Servantie and P. Gaspard, Phys. Rev. Lett 91, 185503 (2003).

[5] J. Servantie and P. Gaspard, Phys. Rev. B 73, 125428 (2006).

[6] Y. Zhao, C.-C. Ma, G.-H. Chen, and Q. Jiang, Phys. Rev. Lett. 91, 175504 (2003).

[7] W. Guo, Y. Guo, H. Gao, Q. Zheng, and W. Zhong, Phys. Rev. Lett. 91, 125501 (2003).

[8] P. Tangney, S. G. Louie, and M. L. Cohen, Phys. Rev. Lett. 93, 065503 (2004).

[9] A. Fennimore, T. D. Yuzvinsky, W.-Q. Han, M. S. Fuhrer, J. Cumings, and A. Zettl, Science 424, 408 (2003).

[10] B. Bourlon, D. C. Glattli, C. Miko, L. Forro, and A. Bachtold, Nano. Lett. 4, 709 (2004).

[11] P. Král and H. R. Sadeghpour, Phys. Rev. B 65, 161401 (2002).

[12] S. Zhang, W. K. Liu, and R. S. Ruoff, Nano. Lett. 4, 293 (2004).

[13] D. W. Brenner, Phys. Rev. B 42, 9458 (1990).

[14] J. P. Lu and W. Yang, Phys. Rev. B 49, 11421 (1994).

[15] A. N. Kolmogorov and V. H. Crespi, Phys. Rev. Lett. 85, 4727 (2000).

[16] C. Daly and J. Krim, Phys. Rev. Lett. 76, 803 (1996).

[17] M. S. Tomassone, J. B. Sokoloff, A. Widom, and J. Krim, Phys. Rev. Lett. 79, 4798 (1997).

[18] K. L. Johnson, K. Kendall, and A. D. Roberts, Proc. R. Soc. Lond. A 324, 301 (1971).

[19] F. P. Bowden and D. Tabor, The Friction and Lubrification of Solids (Oxford Classic Series, 2001). 